\title{
GROUPWARE AND BUSINESS PROCESS IMPROVEMENT: TECHNOLOGY ENABLED ORGANISATIONAL LEARNING
}

\author{
Nereu F. Kock Jr. \\ Robert J. McQueen \\ Department of Management Systems, University of Waikato \\ Private Bag 3105, Hamilton \\ New Zealand \\ E-mails: nfk@waikato.ac.nz., bmcqueen@waikato.ac.nz
}

\begin{abstract}
Much of the evaluative research on groupware in organisations so far has been preoccupied with the role of groupware as a new interaction media to replace or extend face-to-face communication in groups. It has focused on gains and losses from a group interaction point of vicw, typically disregarding the impact of other functions such as allowing public access to relevant historical and business process information concerning the organisation. This paper tries to bridge this gap with a focus on the support provided by groupware to business process improvement groups. This paper is based on a qualitative analysis of the effects of the introduction of an asynchronous groupware system into a service company based in Brazil. That analysis suggests that improvements on business redesign efficiency and effectiveness can be attained not only from asynchronous groupware support to group communication, but also from: 1)Its support for public sharing of historical information aboul former business process improvement; and 2)Its support for providing a repository of information about business processes that could be targets for improvement. The paper is finalised with the proposal of an explanatory model, describing the relationship between the introduction of technology, its integration with a business process improvement meta-process, and its effects on the efficiency and effectiveness of that meta-process.
\end{abstract}

\section{INTRODUCTION}

Organisations can be defined as sets of interrelated business processes, each composed of interacting agents, artifacts, and suppliers whose main goal is to modify inputs in order to produce valuable outputs for customers. As an exchange for delivering outputs to its external customers, the organisation will get an inflow of capital. This capital will typically be invested to supply further demand from customers, in a cyclic and repeatable process (Goldratt and Fox, 1986).

The most successful organisations nowadays are those whose business processes are efficient, i.e. have an optimum balance between the capacity of generating outputs and costs, and effective, i.e. generate outputs that match or exceed the expectations of their customers. This enables those organisations to sustain a balance between production capacity and actual throughput. This balance, however, is hindered by an element being observed since middle 1980s: a continuous and fast changing marketplace (Goldratt and Cox, 1986; Hammer and Champy, 1993), which calls for changes of both radical and continuous proportions (Deming, 1986; Davenport, 1993).

In order to appropriately and quickly respond to external changes, organisations must create local coping mechanisms, at the team level (Senge, 1990). One instance of such decentralised mechanisms is referred to in this paper as business process improvement group (BPIG).

In the following sections business process improvement (BPI) is defined and the importance of BPIGs in a decentralised learning context is discussed. The role of groupware in this context is described and illustrated based on a service organisation's experience. An explanatory model is presented to explain the effects of an instance of groupware - asynchronous groupware - on BPI in organisations. This model is discussed, in the last two sections, in terms of its limitations and implications for future research.

\section{BUSINESS PROCESS IMPROVEMENT}

Business process improvement (BPI) has been regarded as the basis for some world class organisational development approaches (French and Bell, 1990). Two representative examples of succesful BPI endeavours can be found within the management literature. First, the "economic Japanese revolution", strongly based on the concept of gradual process improvement, also referred to as kaizen (Deming, 1986; Walton, 1989). Second, and more recent, the business process re-engineering (BPR) movement, where working groups are expected to propose radical changes for business processes that will, in turn, generate revolutionary quality and productivity improvements (Hammer and Champy, 1993; Davenport, 1993).

BPI is here used as a general term to refer to improvement schemes based on the concept of business process, whether they are radical, incremental, or somewhere in between in terms of degree of improvement sought and realised. BPI is assumed to be carried out by small groups which analyse and propose improvement on business 
processes. From this assumption, two main components are identified as forming the basis on which BPI lies: 1)Business process; and 2)BPI groups.

\section{Business Process}

A business process can be understood as both a value-adding unit and as a set of interrelated activities. As a value-adding unit it is analogous to the classical view of system (Checkland, 1981) for it can be defined as:

An abstract entity which represents the transformation of inputs into outputs. A business process has its suppliers and customers. Inputs are provide by suppliers and the outputs are consumed by customers. The transformation aims at adding value to the inputs by changing them in a way that turns them into desirable outputs from the customers' point of view.

A business process can also be split into other sub-processes. The set of lower level processes into which a business process can be broken are often called "tasks" or "activities" which, in turn, bear some kind of interdependence among themselves (Dennis, Hayes and Daniels, 1994). The main goal for modeling a business process by splitting it into component activities is to understand how the interrelations between activities affect the overall performance of the business process, in terms of efficiency and effectiveness. The combined study of a business process from an activity-based perspective and as a value-adding unit, enables a business process improvement group to propose changes in order to improve some of its attributes such as customer satisfaction, cost, lead time, and simplicity.

\section{Business Process Improvement Groups}

Continuous and radical BPI have often been considered as extremes. This fact has led in the past to the definition of a dual taxonomy (Davenport, 1993), and a continuum of BPI approaches where the main difference is the degree of improvement sought and realised (Dewar and Dutton, 1986; Damanpour, 1988). However, all instances of BPI share some common characteristics, such as their dependence on working groups. These working groups, called here business process improvement groups (BPIGs), have the following properties: 1)They are generally small groups, i.e. from 3 to 12 members (Soles, 1994); 2)Their "modus operandi" is characterised by a process (King, 1990); and 3)They have defined roles (Rosenfeld and Servo, 1990; Hammer and Champy, 1993).

BPIGs are effective tools to enable organisation learning for they foster the thinking on and reshaping of the basic units of organisations, their business processes. In addition, BPIGs have the potential to reduce barriers and foster information sharing among different work teams ${ }^{5}$. This is essential to generate team learning, which has its importance highlighted by Senge (1990) who states: "Team leaming is vital because teams, not individuals, are the fundamental learning unit in modern organizations. This is where the rubber meets the road'; unless teams can leam, the organisation cannot learn" (p. 10).

\section{GROUPWARE AND BUSINESS PROCESS IMPROVEMENT GROUPS}

The term "groupware" is new. In fact it has only become popular since 1991, when PC Week issued the first special supplement ${ }^{6}$ on this subject (McQueen, 1993). Later, in 1992, a conference focusing on commercial CSCW products and their impact on organisations was held in US. That conference was called Groupware ' 92 (Coleman, 1992).

There is no generally accepted definition for groupware. One definition which is acceptable within the scope of this paper is provided as follows. This definition is a convergence of three other definitions (Johansen, 1988; Ellis, Gibbs and Rein, 1991; McQueen, 1993).

Groupware is a generic term for computer-based systems which are particularly used to support groups of people engaged in a common task. Typically, these groups are small and have relevant tasks with definite deadlines.

\footnotetext{
${ }^{5} \mathrm{~A}$ team is a permanent group, who perform activities in a business process. A BPIG is typically a temporary group whose main goal is to improve a business process, whether its members are daily involved with it or not. ${ }^{6}$ PC Week, Special Report on Groupware, October 14, 1991.
} 
BPIGs can have both their efficiency and effectiveness augmented by the use of groupware systems. This assumption is supported by related achievements that have been reported about several aspects, such as:

- Better support to group activities, such as making communication faster and cheaper (Sproull and Kiesler, 1991), reducing paper flow (Wilson, 1991), making filing easier (Brothers et al., 1992) and increasing cross-departmental communication (Clement, 1994).

- Positive effects on individual behaviour, such as reducing stress (Pietro, 1992) and making individuals communicate more openly (Sproull and Kiesler, 1991).

- Positive effects on group behaviour, such as distributing individual contributions more evenly (Nunamaker et al., 1991), separating ideas from individuals (Chidambaram and Kautz, 1993), reducing repetition of old ideas and increasing commitment with group decisions (Sheffield and Gallupe, 1993).

Despite the broad range of possible applications of groupware systems to support BPIGs, most of the research has been focused on group decision support systems. These systems are considered a category of groupware. They usually support decision meetings, where the final decision is the main outcome. Brainstorming, voting, ranking and classification of ideas are examples of tasks supported by a typical group decision support system (Dennis, Hayes and Daniels, 1994).

Group decision support systems support synchronous communication, where users must be connected to the system at the sanne time to be able to interact. It is like the telephone, in which one cannot communicate to another person unless $\mathrm{s} / \mathrm{he}$ is able to answer the call. This category of groupware systems is opposed to asynchronous groupware, where individuals can interact at different times. Examples of asynchronous groupware are electronic-mail, computer conferencing and workflow control systems (Kock, McQueen and Fernandes, 1994).

\section{GROUPWARE AND BPI: A SERVICE COMPANY'S EXPERIENCE}

The experience of a service company based in Brazil is investigated in this section. This investigation aims at illustrating the concept of BPI groups and related effects of groupware technology. The service company studied is an international events organiser, referred to as Equipe ${ }^{7}$.

Equipe was started by early 1964 as a consulting firm. Its main goal was to deliver consulting services to the manufacturing industry. Equipe's main activity in the early years after establishment was facilities layout planning. After approximately five years struggling to survive amongst a fierce competition, they glimpsed a new market niche, to organise the participation of Brazilian companies in international fairs in order to promote their products in new markets. It became and remained Equipe's main business. Soon after having started in the new market niche of international events operation, Equipe had built a strong reputation of service quality and reliability. Equipe sustained the position of main supplier in Brazil for international events held in Europe, United States and Asian countries for 20 years, led by a couple of aggressive executives. Some dark clouds, however, showed up on the horizon by 1989.

\section{Inability to Learn and Adapt to Change}

From early 1989 on, the events industry client basis started to shrink in Brazil. New competitors entered the market grounded on either vertical integration of stands manufacturing and rental, or strong partnerships with stand suppliers. As most of the costs of an exposition come from the design, rent, transport and assembly of stands, it seemed to be impossible for Equipe to survive in this new competitive arena without good partnerships. Prices would increase and quality deteriorate without it. However, Equipe's main executives failed to grasp the competitive importance of partnerships, mainly because they were too busy trying to find new clients and cope with an increasingly changing marketplace. As one of them mentioned with a frown of concern on his face: "We're passing through a period of turbulence. I hope things will settle down after a while".

Equipe's executives centralised most of the decisions. This approach for a growing company in a fierce competing environment proved to be a disaster in several occasions. One example was the insistence of Equipe's executives on developing software systems at home to support their operations. This position was maintained against the advice of consultants and internal computer support staff, mainly based on fear that the company's know-how on events operation would go public if Equipe hired an external software development

\footnotetext{
${ }^{7}$ It is a fictitious name. The real name is concealed to protect confidentiality.
} 
company. This drove the company into a project that took three years to develop a computer system, which turned out to present several limitations. The system was designed to support the integrated work carried out by the Marketing and Operations divisions. Internal users of the system would basically be able to store updated information regarding customers and keep track of the evolution of respective services provided by Equipe through stages, ranging from prospecting new customers to post-evaluation of events. After two years the system finally achieved an acceptable level of efficiency, but now new technologies would enable radical changes in the core processes. These changes called for deep modifications in the system, which was eventually abandoned.

Several interviews revealed symptoms of an organisation in which the learning process was centered on its top management. Both senior executives, who shared the CEO position, showed clear signs of stress. They were frustrated with the new market situation, accusing some stand suppliers of lack of loyalty. The failure of the software system described above was a source of cynicism and lack of trust on information technology. Employees were unmotivated, reporting as the most important reason for their lack of motivation the excessive centralisation of decisions by the two executives. The employees showed no interest in improving business processes, and a strong lack of interest on learning new methods and techniques. As one of the managers reported: "What keeps them working are their wages and the prospect of international trips".

\section{Starting the Business Process Improvement Groups}

The inability to motivate workers at Equipe cailed for changes in the management approach. Some techniques for enhancing the level of participation in decisions were tested such as brainstorming sessions, suggestion boxes, and campaigns for new ideas. None of them generated the expected gains, basically due to two reasons. First, they were strictly based on the assumption that front-line workers should have an active role on management and solution of problems, which is one of the several facets of process improvement. Employees were, among other things, called to participate in routine strategic decisions irrespective of the type of decisions, following an approach suggested by Semler $(1989 ; 1993)$. However, it proved to be a counterproductive strategy, supporting the assumption that group decisions are not necessarily better than individual decisions. In fact, they can cause decisions to take more time to be reached and also be of less quality (Senge, 1990). Strategic decisions to form new partnerships or take a large government contract, for example, were found, after some trials, better be made only by managers as usual. Sharing the responsibility of taking decisions with large groups of employees not directly involved with decision making in a daily basis simply delayed what could not be delayed, undermining both employees and management's confidence on participatory management. Second, business processes received low priority. For example, the whole set of interrelated activities involved in signing a new contract, from gathering relevant customer and event information to the analysis of contribution margins, was never discussed. The emphasis was on having employees participate in management activities, rather than analysing how activities were executed and improvements could be attained. New ideas coming from employees covered a broad range of subjects, from new designs for fliers to better wages. The broad range of subjects and the repetition of ideas progressively undermined the interest of managers, and consequently employee motivation to generate new ideas.

One of the authors (Kock) worked as a organisational consultant at Equipe for a little over one year, which enabled him to perform a study with action research characteristics (McKernan, 1991; Elden and Chisholm, 1993). The work was started with an analysis of the organisation and proposal of a set of changes at the management and operational levels. Based on former experience and on a methodology developed to implement group-based quality and productivity improvement (Kock and Tomelin, 1994), a project to implement BPIGs was planned and put into practice.

It was predetermined that all BPIGs at Equipe should complete a process improvement proposal within a limited time. As opposed to business process re-engineering groups (Hammer and Champy, 1993; Soles, 1994), they were neither expected to be composed mostly by management staff and external consultants, nor to come up with genuinely radical improvements - i.e. the improvements proposed could be modest and gradual. Each BPIG was expected to complete the analysis and redesign of a business process within one to eight weeks. After that a proposal should be generated and handed to an improvement committee (which included the researcher) to be evaluated. Should the proposal be considered attainable and prone to generate return on investment, the improvement committee would coordinate with the appropriate staff to implement the proposed redesign.

\section{Introduction of an Asynchronous Groupware System}

BPIGs started in a very modest fashion. After three months eight BPIGs have been completed and five were ongoing. As the company had nearly 70 employees, its full capacity would be approximately 10 to 23 BPIGs at 
a time, what would give an average of 33 groups per quarter ${ }^{8}$. Five, out of the first eight BPIGs, proposed business process improvements. The remaining three proposed minor problem oriented solutions which affected business processes but clearly were not based on business process analysis, i.e. they were based on problem analysis rather than on business process analysis ${ }^{9}$, which is consistent with the results of previous research (Dennis et al., 1993). This happened despite the eight hours training session in which the concept of business process was discussed along with a structured approach to perform analysis and design. These training sessions were performed with groups of 10 to 20 workers and involved all company members, including top management.

Some employees who have taken part in no improvement group reported, as one of the causes for such a behaviour, that they could not attend to BPIG meetings because they would likely to be engaged in an external activity (e.g. discussing a contract at a client) during group meetings. This problem called for an asynchronous groupware system through which group members could interact from their own computers at different times. As nearly $95 \%$ of the workers had access to a networked computer, an electronic-mail system was installed and adapted to incorporate simple computer conferencing features. Each BPIG was assigned a group mailbox upon its creation. BPIG mailbox titles were suggestive of the business process under consideration, and held postings from group members related to different stages of the BPI meta-process (i.e. business process definition, analysis, and redesign). Some BPIG mailboxes allowed public access within the organisation, so other employees could participate on ongoing discussions. Other BPIG mailboxes restricted access to their respective BPIG members only, when the BPI work was perceived as addressing delicate matters.

Some public mailboxes were created and gradually fed with information regarding the main business processes of the organisation. This information embodied descriptions of external suppliers for the process, main activities, people and resources involved, and main customers of the process. It also contained some regularly updated postings with information about efficiency, costs, lead time, and customer complaints for each business process. Employees were given some training on how to use the groupware system to carry out BPIG activities and were encouraged to make as much use of the system as possible.

\section{Qualitative Results From the Use of the System}

During the first three months after the introduction of the groupware tool the number of BPI groups nearly doubled. The percentage of groups proposing process focused improvements remained the same, relatively to the total numbers of groups, but a help document attached as a message in a public mailbox was reported as a useful support for the members to avoid lack of structure when carrying out BPIG activities. From a communication media perspective, most of the groups reported using the groupware system for supporting approximately two thirds of the interactions between group members.

Some problems arose at the earlier stages of utilisation of the groupware system. These problems were caused by the lack of features presented by asynchronous groupware to replace face-to-face meetings. Each BPIG had a leader and a facilitator, chosen from within the group by the group members themselves. The leader basically coached the group, while the facilitator provided support on business process modeling and analysis. In a typical face-to-face meeting all tasks are accomplished by all members at the same time. Through the use of asynchronous groupware, though, it was not possible or would take too long for them to communicate, since members are separated by time and space. It called for a change in the structure of the traditional BPIG.

The new structure devised pushed the responsibility for accomplishing each one of the stages of a BPIG to a certain member of the group, which would post in the mailbox the results of her/his work and wait for comments. For example, the analysis stage, in which the business process is modeled and analysed, is carried out by one of the members supported electronically and face-to-face by the facilitator. The function of the leader is still to coordinate the activities and resolve conflicts. Both the leader and the facilitator functions can be overlapped with other group activities, e.g. process analysis. Members reported that this new structure improved productivity. Reasons presented were the ability to carry out parallel activities and the reduction of the time spent, in comparison to ordinary meetings.

Other results reported during interviews or identified via participant observation were: 1)The reduction of redundant improvement proposals after the adoption of the new BPI approach, when compared with previous improvement approaches tried at Equipe. 2)The perceived higher efficiency in the analysis of business processes, reported as the most time consuming BPI stage, and in the redesign stage. 3)The perceived high importance of the BP analysis stage to the next stage, BP redesign. 4)The perceived usefulness of the public

\footnotetext{
${ }^{8}$ Considering that a group would take in average 4 weeks to complete its work.

${ }^{9}$ For example, the response to customer complaints on quotations for events was a change in the format of the quotation document, as opposed to a change in the way the quotation process was carried out.
} 
access to historical information on former BPI, both as a source of examples to perform current BPI and as a source of solutions whose application do not demand duplicate BPI effort. 5)The perceived value of public access to business process information regarding customer satisfaction, throughput, and lead time. It was reported that the easy access to this information allowed better identification of BPI opportunities and performance in the BP analysis. As reported by one events operator "Before we started using it [the groupware system] we had to spend hours analysing a pile of paper to find out some information about our main operations. Even so I couldn't tell you whether there was something wrong with them or not". 6)The perceived value of faster and easier communication supported by the system, in the BP analysis and design stages, for the identification and correction of mistakes and wrong assumptions. 7)The perceived importance of the role of the groupware system to support the spread of historical information about former BPI and BP-related information. Both senior executives reported great satisfaction with the results of the BPIGs. One of them reported that "We have never been through a so successful motivational endeavour since the firm was founded". It was also reported that BPIGs improved the relationship between high management staff, middle managers, and workers. Some managers reported being impressed, when browsing through BPIG mailboxes held by the groupware system, by the increased awareness of workers about the problems of the firm and their willingness to find solutions. After one international event where some problems were detected at the check-in and stand assembly processes, groups were immediately formed and came up with effective business process redesigns. This was a hint that the ability to learn and adapt to change was spreading over the company's body, instead of concentrating only in its head.

\section{AN EXPLANATORY MODEL}

The analysis of Equipe's experience, complemented by a review of related literature, provides the necessary background to build an explanatory model regarding the use of asynchronous groupware to support business process improvement. This model is illustrated in Figure 1.

In Figure 1, each rectangle represents one variable. Each arrow represents an interdependence between variables. At the destination of each arrow is a variable which depends on the variable at the source of the arrow. In this sense, for example, the variable $<$ BPI framework $>$ is affected by the variables <BPI metaprocess $>$ and $<$ Asynchronous groupware methodology $>$. These two variables are also the independent variables of the model, since they are not influenced by any variable. The dependent variables of the model, seen as a whole, are the two rightmost ones <BPI efficiency> and <BPI effectiveness $>$. The variables in between have the function of providing an explanation on how the model operates.

The model depicted in Figure 1 places emphasis on the interdependence between variables. A description for each one of the variables is provided as follows, starting from the right part of the figure and moving leftwards. The meaning of the interdependencies is supplied along with supporting rationale based on Equipe's experience and related literature.

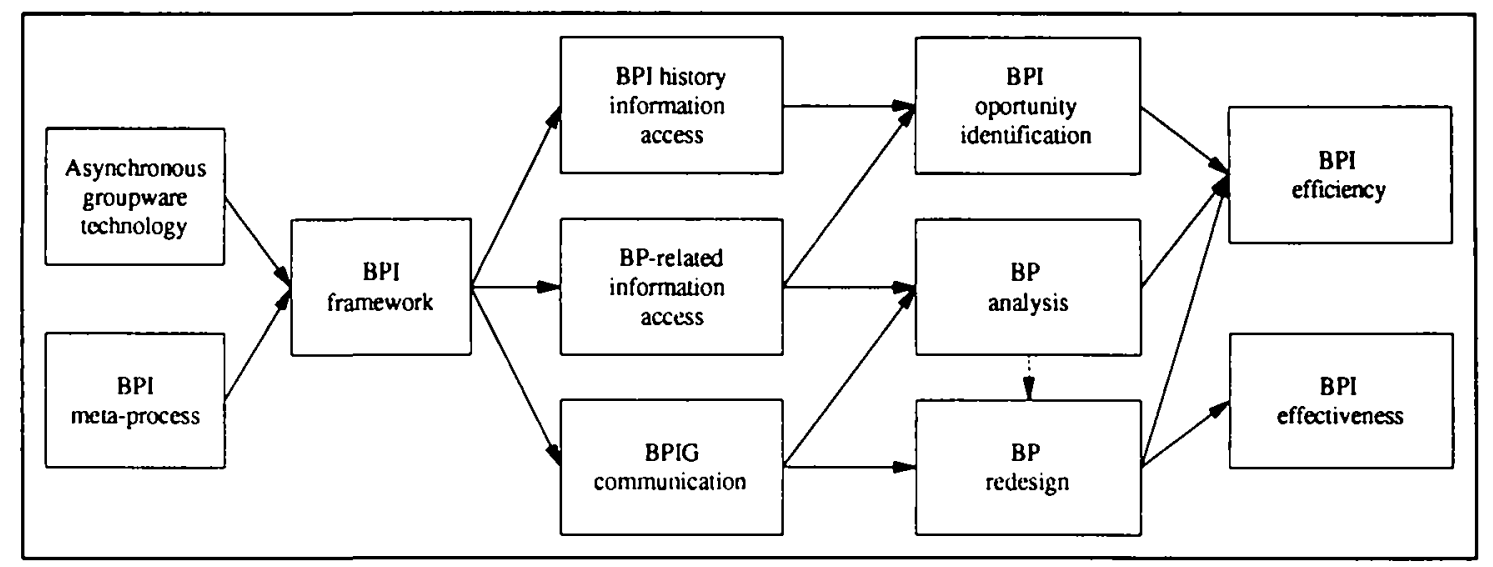

Figure 1: Asynchronous Groupware and Business Process Improvement (An Explanatory Model)

- BPI efficiency. This variable represents the efficiency of the BPI meta-process. It is a combination of the measures of the average time spent by BPIGs to redesign a business process, the number of BPIGs at a specific time, and the costs involved in the BPI meta-process. 
- BPI effectiveness. It represents the average effectiveness of the business process redesigns carried out by BPI groups. This effectiveness can be understood as the effect that the redesign has on the quality and productivity of the redesigned business process. BPI effectiveness can be evaluated by experts, who forecast quality and productivity improvements in the business process after the redesign. However, an actual improvement measure can only be obtained after the redesign is consolidated through implementation and routinisation.

- BPI opportunity identification. This variable represents both the efficiency and effectiveness in the identification of BPI opportunities. By making it easier, less expensive, or more efficient - e.g. by providing information about similar BPI that can be replicated with little effort - the efficiency of the BPI metaprocess itself is increased, which is illustrated by the connection between this variable and <BPI efficiency>. This assumption is suggested by the reduction in the number of redundant improvement ideas, reported from previous improvement approaches tried at Equipe, after the new BPI framework was implemented.

- BP analysis. This variable represents both the efficiency and effectiveness of the analysis stage of BPI, performed by a group. Measurements for this variable can be done based on the time taken to carry out the $\mathrm{BP}$ analysis, as well as on the clarity and completeness of the information generated for the next stage in the BPI meta-process, BP redesign. Variations in the $<\mathrm{BP}$ analysis $>$ variable affect the efficiency of the whole BPI meta-process as well as the next stage in the BPI meta-process, BP redesign. The effect on the efficiency of the BPI meta-process is suggested by the fact that it is the longest stage of the meta-process. The effect on the next stage, BP redesign, was indicated in interviews with several BPIGs members, where they showed concern about the negative effects of lack of clarity and completeness in the information coming from the BP analysis stage.

- $B P$ redesign. This variable represents both the efficiency and effectiveness of the redesign stage of BPI, performed by a group. This variable is likely to affect both the efficiency and effectiveness of the BPI meta-process as a whole. As it is a substantial part of the BPI meta-process, a loss in efficiency in the BP redesign stage - e.g. extended time and high costs - has direct impact on BPI's efficiency. On the other hand, a poor redesign in terms of quality is likely to impair the effectiveness of the BPI meta-process, as the actual benefits sought may not be attained. The connection between efficiency and effectiveness of the BP redesign and the BPI meta-process is supported by a number of previous studies (Hammer and Champy, 1993; Davenport, 1993; Dennis, Hayes and Daniels, 1994). Yet metrics to predict the effectiveness of BPI meta-processes, based on BP redesign quality are still to be developed and validated.

- BPI history information access. This variable represents the degree of access prospective BPIG members have to historical information about former BPI. This historical information consists of documentation generated by former business process improvement groups. A high degree of access to information regarding other BPIGs' work is likely to positively affect the variable <BPI opportunity identification>. It provides examples to be followed, and results that can be replicated without duplicated effort, as reported by BPIG members at Equipe.

- BP-related information access. This variable represents the degree of access prospective and actual BPIG members have to information regarding business processes. It affects two variables: $<$ BPI opportunity identification $>$ and $<\mathrm{BP}$ analysis $>$. The access to BP-related information, such as customer satisfaction and throughput, allows prospective BPIG members to identify the best opportunities for BPI. It also improves the productivity of business process analysis, which relies heavily on information gathering and analysis. These effects were reported by BPIG members at Equipe.

- BPIG communication. This variable represents the efficiency and effectiveness of communication between the members of a BPIG and with outside. It is likely to affect both the efficiency and effectiveness of the BPI analysis and design stages, i.e. the variables $<\mathrm{BP}$ analysis $>$ and $<\mathrm{BP}$ design $>$. This relationship is explained by the fact that the analysis and redesign phases rely strongly on information exchange and discussion between BPIG members and with others who, despite being outside the group, can provide relevant information about the business process being improved and possible redesign alternatives. This point is supported by the literature on group work (Napier and Gershenfeld, 1993) and also by Equipe's experience. The experience at Equipe shows that fast and easy communication between BPIG members 
and with others in the organisation, makes it easier for mistakes in the BP analysis and redesign stages to be identified, which affects both the efficiency and effectiveness of those stages.

- BPI framework. This variable represents the existence of a BPI framework. This framework is the result of the combination between a BPI meta-process, i.e. a structured process to perform business process improvement, and asynchronous groupware technology, i.e. a software system with asynchronous groupware features. The BPI framework will allow business process improvement to be carried out with technology, which directly affects three variables: <BPI history information access>, <BP-related information access $>$, and <BPIG communication>. From the analysis of Equipe's experience it can be seen that the introduction of an asynchronous groupware system is unlikely, per se, to impact business process improvement in a positive way. It must be accompanied by a redesign of the meta-process through which BPI is carried out. However, Equipe's experience also suggests the potential of asynchronous groupware systems to improve organisational access to BPI historical information and BP-related information access. The effect of asynchronous groupware on BPIG communication is extensively suppored by the literature, as discussed at the end of this section.

- Asynchronous groupware technology. This variable represents the presence of a software system with asynchronous groupware features, which is integrated with the BPI meta-process to form the BPI framework.

- BPI meta-process. This variable represents the existence of a BPI meta-process to perform business process improvement. It is integrated with the asynchronous groupware system to generate the BPI framework.

The literature of evaluative studies of groupware systems to support communication in groups generally reports gains in productivity and quality (to a lower extent), concerning the interaction process (Sproull and Kiesler, 1991; Nunamaker et al., 1991; Brothers et al., 1992; Chidambaram and Kautz, 1993; Clement, 1994). Support to those results comes from Equipe's experience, specifically regarding asynchronous groupware, by the fact that some group members could not participate on BPIGs, before the introduction of the groupware system, due to external meetings and time constraints. After the introduction of the system they could become fully engaged members of BPIGs, and reported enjoying a more active participation in the improvement of the company.

The proposed explanatory model, though, presents a contrast with much of the research performed so far on groupware evaluation. Former research has been preoccupied with the role of groupware as a tool to replace or expand face-to-face communication in groups (Serida-Nishimura, 1994). It has focused on gains and losses from a group interaction point of view and, with some exceptions (Orlikowski, 1992; Rein, Holsapple and Whinston, 1993), typically disregarded the impact of other groupware functions such as allowing public access to relevant historical and business process information regarding the organisation. These latter are the main aspects stressed in the model described in this paper.

\section{LIMITATIONS OF THE CURRENT RESEARCH}

The results outlined in the explanatory model might have been distorted by some sources of bias. The two main sources of bias in this research were: 1) The fact that the research was based on only one site, which may affect the generality of the research from an organisational perspective, and 2)The high involvement of the researcher with the organisation, conducting the research concurrently with a consulting project. In fact, both of the sources of bias are characteristic of action research (Galliers, 1992: Sommer, 1994). Most action research in organisations have been performed in only one or a few sites in order to allow the researcher to have a deeper understanding of the context being studied within a relatively short period of time (Candlin and Wright, 1991). Several sites would either delay the reporting of results, or disperse the focus of the research. Both effects are undesirable. The second source of bias, the high involvement of the researcher, was inevitable since it is one of the attributes that define action research as a distinctive research approach (Francis, 1991).

The first source of bias could be reduced in the current study by following one of two different options. The first option would be to produce as the final result of the research a specific model, explaining the relationship between asynchronous groupware and BPI within a specific context (e.g. a type of organisation or activity). The second option, which was the one adopted, was to base the investigation on units of analysis that are common to all organisations, i.e. business process, business process agents, business process improvement, and business process improvement groups. It also allowed the access to a considerable number of instances for each unit of analysis, which is likely to improve the significance of the results. 
The second source of bias could also be reduced by the researcher following two complementary protocols: 1)Analyse data in a rational way and from a disengaged perspective. This is highly dependent on the researcher's own ability to detach from the context being studied, and on his/her lack of commitment toward positive results for the organisation. 2)Involve a group of external researchers in the analysis of the data collected. In this research none of the protocols were fully implemented. The breach in the first protocol was caused by the position hold by the researcher as a coach for some BPIGs and champion in the introduction of the groupware system. This position was taken in order to enhance the likelihood of success of the endeavour. It could have affected the results of the opinions expressed in the interviews, as the interviewees could feel compelled to express over-optimistic opinions about the effect of the system in order to please the researcher. An alternative to reduce this source of bias would be the search of coaches and champions for the research project within the organisation. This alternative, though, could hinder the research development due to lack of suitable individuals with enough time to commit to the research project. The breach in the second protocol is due to the fact that the study was carried out by only one researcher, in cooperation with some of the organisation members. Since there were potential sources of bias from both the researcher and organisation members, an alternative would be to involve researchers from outside the organisation.

\section{RECOMMENDATION FOR FURTHER RESEARCH}

The explanatory model proposed is preliminary. It is the result of a qualitative analysis based on a study with action research characteristics. Further research is suggested to improve the model proposed by concurrently expanding and refining it. Possible research approaches suggested are case research (Yin, 1989) and action research (Checkland, 1991). Other major research approaches such as survey research and experimental research are not recommended due to some constraints, such as: 1)The longitudinal property of the research that originated the model, and its disparity with cross-sectional studies; 2)The limited number of organisations currently using asynchronous groupware systems to support BPI; and 3)The orientation of the model towards the description of real organisational settings, as opposed to controlled environments.

\section{ACKNOWLEDGMENTS}

The authors would like to thank the anonymous reviewers of this paper for their helpful comments. The authors also reported on this study in "Integrating Groupware into a Business Process Improvement Framework" in Information Technology \& People, Vol. 8, No. 4, 1995.

\section{REFERENCES}

Brothers, L., J. Hollan, J. Nielsen, S. Stornetta, S. Abney, G. Furnas and M. Littman (1992), Supporting Informal Communication Via Ephemeral Interest Groups, Proceedings of CSCW'92 Conference, J. Turner and R. Kraut (Eds), New York: ACM Press, pp. 84 to 90.

Candlin, D.B. and S. Wright (1991), Managing the Introduction of Expert Systems, International Journal of Operations \& Production Management, Vol.12, No.1, pp. 46-59.

Checkland, P. (1981), Systems Thinking, Systems Practice, New York: John Wiley \& Sons.

Checkland, P. (1991), From Framework through Experience to Learning: the essential nature of Action Research, Information Systems Research: Contemporary Approaches and Emergent Traditions, H. Nissen, H.K. Klein and R. Hirschheim (Eds), New York: North-Holland, pp. 397-403.

Chidambaram, L. and J.A. Kautz (1993); Defining Common Ground: Managing Diversity Through Electronic Meeting Systems, Proceedings of the 14th International Conference on Information Systems, J.I. Degross, R.P. Bostrom and D. Robey (Eds), New York: The Association for Computing Machinery, pp. 1-11.

Clement, A. (1994), Computing at Work: Empowering Action by Low-level Users, Communications of ACM, Vol.37, No.1, pp. 53-63.

Coleman, D. (Ed) (1992), Proceedings of Groupware '92, San Mateo, CA: Morgan Kaufmann, pp. 84-90.

Damanpour, F. (1988), Innovation Type, Radicalness, and the Adoption Process, Communications Research, Vol.15, pp. 545-567.

Davenport, T.H. (1993), Process Innovation, Boston: Harvard Business Press.

Deming, W.E. (1986), Out of The Crisis, MIT, Cambridge, MA: Center for Advanced Engineering Study.

Dennis, A.R., R.M. Daniels Jr, G. Hayes, J.F. Nunamaker Jr. (1993), Methodology-Driven Use of Automated Support in Business Process Re-engineering, Journal of Management Information Systems, Vol.10, No.3, pp. 117-138. 
Dennis, A.R., G.S. Hayes and R.M. Daniels Jr. (1994), Re-engineering Business Process Modeling, Proceedings of 27th Hawaii International Conference on System Sciences, Los Alamitos, CA: IEEE Computer Society Press, pp. 244-253.

Dewar, R.D. and J.E. Dutton (1986), The Adoption of Radical and Incremental Innovations: An Empirical Analysis, Management Science, Vol.32, pp. 1422-1433.

Elden, M. and R.F. Chisholm (1993), Emerging Varieties of Action Research, Human Relations, Vol. 46, No. 2, pp. 121-141.

Ellis, C.A., S.J. Gibbs and G.L. Rein (1991), Groupware: Some Issues and Experiences, Communications of ACM, Vol. 34, No. 1, pp. 38-58.

Francis, D. (1991), Moving from Non-Interventionist Research to Participatory Action, Proceedings of The First World Congress on Action Research, Vol.2, C. Collins \& P. Chippendale (Eds), Sunnybank Hills, Queensland: Acom, pp. 31-42.

French, W.L. and C.H. Bell Jr. (1990), Organization Development, Englewood Cliffs, NJ: Prentice Hall.

Galliers, R. (1992), Choosing Information Systems Research Approaches, Information Systems Research, R. Galliers (Ed), Boston: Blackwell Scientific Publications, pp. 144-162.

Goldratt, E.M. and J. Cox (1986), The Goal: A Process of Ongoing Improvement, New York: North River Press.

Goldratt, E.M. and R.E. Fox (1986), The Race, New York: North River Press.

Hammer, M. and Champy, J. (1993), Reengineering the Corporation, New York: Harper Business.

Johansen, R. (1988), Groupware: Computer Support for Business Teams, New York: The Free Press.

King, N (1990), Innovation at Work: The Research Literature, Innovation and Creativity at Work, M.A. West and J.L. Farr (Eds), New York: John Wiley \& Sons, pp. 15-59.

Kock Jr., N.F., R.J. McQueen and C.T. Fernandes (1994), Quality Management and Groupware, Proceedings of the 18th Annual Conference of the National Association of Higher Education Programmes in Management, Information Management Section, C.M. da Silva, J.E. Fensterseifer and C.G. Neto (Eds), Rio de Janeiro: ANPAD, pp. 98-113.

Kock Jr., N.F. and C.A. Tomelin (1994), PMQP: Total Quality Management in Practice, Curitiba, Brazil: Business Development Centre-SENAC/PR (in Portuguese).

McKernan, J. (1991), Action Research, Proceedings of The First World Congress on Action Research, Vol.2, C. Collins \& P. Chippendale (Eds), Sunnybank Hills, Queensland: Acorn, pp.65-86.

McQueen, R.J. (1993), Groupware: Experience in New Zealand, Proceedings of the 13th New Zealand Computer Society Conference, Vol.1, Auckland, New Zealand: The New Zealand Computer Society, pp. 10-20.

Napier, R.W. and M.K. Gershenfeld (1993), Groups Theory and Experience, Boston: Houghton Mifflin. Nunamaker, J.F., A.R. Dennis, J.S. Valacich, D.R. Vogel, and J.F. George (1991), Electronic Meeting Systems to Support Group Work, Communications of ACM, Vol.34, No.7, pp. 40-61.

Orlikowski, W.J. (1992), Learning from Notes: Organizational Issues in Groupware Implementation, Proceedings of CSCW'92 Conference, J. Turner and R. Kraut (Eds), New York: The Association for Computing Machinery, pp. 362-369.

Pietro, C. (1992), Groupware Meetings that Work, Proceedings of Groupware'92 Conference, San Mateo, CA: Morgan Kaufmann, pp. 50-58.

Rein, G.L., C.L. Holsapple and A.B. Whinston (1993), Computer Support of Organisation Design and Learning, Journal of Organizational Computing, Vol.3, No. 1, pp. 87-120.

Rosenfeld, R. and J.C. Servo (1990), Facilitating Innovation in Large Organizations, Innovation and Creativity at Work, M. A. West and J. L. Farr (Eds), New York: John Wiley \& Sons, pp. 251-263.

Semler, R. (1989), Managing Without Managers, Harvard Business Review, September-October, pp. 76-84.

Semler, R. (1993), Maverick, London: Arrow.

Senge. P.M. (1990), The Fifth Discipline, New York: Doubleday.

Serida-Nishimura, J.F. (1994), An Organizational Culture Pespective for the Study of Group Support

Systems, Proceedings of the 15th Intemational Conference on Information Systems, J.I. Degross, S.L. Huff and M.C. Munro (Eds), New York: The Association for Computing Machinery, pp. 201-211.

Sheffield, J. and B. Gallupe (1993), Using Electronic Meeting Technology to Support Economic Policy Development in New Zealand: Short Term Results, Journal of Management Information Systems, Vol.10, No.3, pp. 97-116.

Soles, S. (1994), Work Reengineering and Workflows: Comparative Methods, The Workflow Paradigm, E. White and L. Fischer (Eds.), Alameda, CA: Future Strategies.

Sommer, R. (1994), Serving Two Masters, The Journal of Consumer Affairs, Vol.28, No.1, pp. 170-187. 
Sproull, L. and S. Kiesler (1991), Computers, Networks and Work, Scientific American, September, pp. 8491.

Tapscott, D. and A. Carston (1993), Paradigm Shift, New York: McGraw-Hill.

Walton, M. (1989), The Deming Management Method, London: Mercury.

Wilson, P. (1991), Computer Supported Cooperative Work, Oxford, England: Intellect.

Yin, R.K. (1989), Research Design Issues in Using the Case Study Method to Study Management Information Systems, The Information Systems Research Challenge: Qualitative Research Methods, J.I. Cash and P.R. Lawrence (Eds), Boston: Harvard Business School, pp. 1-6. 